\title{
MODELO DE DISTRIBUCIÓN ESPACIAL, TEMPORAL Y DE ESPECIACIÓN DEL INVENTARIO DE EMISIONES DE MÉXICO (AÑO BASE 2008) PARA SU USO EN MODELIZACIÓN DE CALIDAD DEL AIRE (DiETE)
}

\author{
José Agustín GARCÍA-REYNOSO*, Bertha Eugenia MAR-MORALES y Luis Gerardo RUIZ-SUÁREZ
}

Centro de Ciencias de la Atmósfera, Universidad Nacional Autónoma de México, Circuito de la Investigación Científica s/n, Ciudad Universitaria, 04510 Ciudad de México, México

*Autor para correspondencia: agustin@atmosfera.unam.mx

(Recibido febrero 2017; aceptado marzo 2018)

Palabras clave: contaminación atmosférica, emisiones, distribución horaria, distribución geográfica, distribución especies químicas

\section{RESUMEN}

Este trabajo presenta el modelo de distribución espacial, temporal y de especiación (DiETE) del Inventario de Emisiones de México para el año base 2008. Se describen tanto la metodología utilizada como la información empleada para realizar la distribución espacial, temporal y química de las emisiones. Se muestran los resultados de la aplicación de este modelo al Inventario Nacional de Emisiones de México (INEM) 2008 y la validación del procedimiento. El INEM 2008 considera siete contaminantes (compuestos orgánicos volátiles [COV], amoniaco $\left[\mathrm{NH}_{3}\right]$, óxido de nitrógeno [NOx], dióxido de azufre $\left[\mathrm{SO}_{2}\right.$ ], monóxido de carbono [CO], y partículas $\mathrm{PM}_{10}$ y $\mathrm{PM}_{2.5}$ ) emitidos por tres tipos de fuentes: fijas, de área y móviles, las cuales se subdividen en 258, 64 y 17 categorías, respectivamente. A cada categoría se le relacionó con un código de clasificación de fuente (SCC, por sus siglas en inglés). Los perfiles de tiempo y especiación química se obtuvieron a partir del SCC de cada categoría. Se identificó que la masa total de los COV obtenidos con este modelo es hasta $17 \%$ menor que el valor del inventario. El DiETE puede generar archivos para los mecanismos RADM2, RACM, CBM5 y SAPRC99, que emplea el model WRF-chem. Si bien se han utilizado modelos de emisiones en otros estudios, éste es el primero que incluye la validación en el procedimiento de conversión. Es útil para todo el año y se puede emplear para la evaluación de medidas mediante la modelización en la gestión ambiental.

Key words: atmospheric pollution, emissions, hourly distribution, geographical distribution, chemical species distribution

\begin{abstract}
This paper presents the spatial, temporal and speciation distribution model (DiETE) of the 2008 Mexico National Emissions Inventory (MNEI). The methodology and information used to estimate the spatial, temporal and chemical distribution of the emissions is described. The results and validation procedure are shown using the 2008 MNEI, which considers seven pollutants: volatile organic compounds (VOC), ammonia $\left(\mathrm{NH}_{3}\right)$, nitrogen oxide $(\mathrm{NOx})$, sulfur dioxide $\left(\mathrm{SO}_{2}\right)$, carbon monoxide $(\mathrm{CO}), \mathrm{PM}_{10}$ and
\end{abstract}


$\mathrm{PM}_{2.5}$. Three source types are also considered: fixed, area and mobile. They are further subdivided into 258, 64 and 17 categories, respectively. Each category was related to a source classification code (SCC). Temporal and chemical profiles were obtained from each SCC category. It was identified that the total mass of VOC in the aggregation procedure compared to the emission inventory produces a difference up to $17 \%$. The DiETE model can generate files for RADM2, RACM, CBM5 and SAPRC99, used by WRF-chem. Although emissions models have been used in other studies, this is the first to include validation in the conversion procedure, which is useful for the whole year and can be used for environmental management.

\section{INTRODUCCIÓN}

La contaminación atmosférica puede inducir mortalidad prematura (Goldstone 2013), por lo cual es importante evaluar y reducir la exposición de la población a altas concentraciones de contaminantes mediante medidas de control. Los modelos de calidad del aire (MCA) se emplean para construir mapas de valores medios o máximos de contaminantes, o mapas de exposición y excedencias de niveles críticos. Se usan en el proceso de gestión ambiental para la selección y evaluación de políticas ambientales de acuerdo con la evaluación de los costos y beneficios de la aplicación de éstas (Qiu et al. 2015), con el objetivo de reducir la exposición poblacional a contaminantes ambientales.

Los MCA son un conjunto complejo de modelos matemáticos que se encuentran acoplados (Grell et al. 2005) para simular numéricamente las concentraciones de contaminantes, su transporte meteorológico y sus transformaciones fisicoquímicas, usando como insumo fundamental las emisiones, cuya distribución espacial y temporal asume supuestos u observaciones sobre las actividades humanas (económicas, recreativas, de transporte, etc.).

Los inventarios elaborados por las autoridades ambientales poseen una resolución espacial y temporal que impide su utilización en modelización de calidad del aire. Por lo tanto, se requiere de información y procesamiento adicional para convertir un inventario típico en un archivo útil para la modelización; de ahí la importancia del modelo de emisiones propuesto en este documento, el cual distribuye las emisiones que reporta el Inventario Nacional de Emisiones de México (INEM) en el territorio mexicano, en el tiempo y en las especies requeridas para la modelización.

Se han presentado diferentes trabajos de modelización de calidad del aire para la zona centro de México (García-Reynoso et al. 2009, Mena-Carrasco et al. 2009, Zavala et al. 2009, Zhang y Dubey 2009, Zhang et al. 2009, Song et al. 2010, Jazcilevich et al. 2011, Almanza et al. 2014, García-Escalante et al. 2014, Ruiz-Suárez 2015) y para toda la República Mexicana (Tie et al. 2007, Emmons et al. 2010). En el desarrollo de dichas investigaciones se han empleado inventarios de emisiones. Por otra parte, se han realizado trabajos en que se muestra el procesamiento de emisiones para la Ciudad de México (García-Reynoso 2002, Ortiz 2005, Guevara et al. 2017), los cuales se emplean para la modelización de calidad del aire.

Sin embargo, ninguno de los trabajos mencionados presenta el procedimiento de generación del inventario para modelización en todo México, con verificación de las emisiones en cada parte del proceso, como en este documento, el cual además describe el método utilizado para convertir el inventario anual (en formato tabulado de magnitudes totales) a un inventario utilizable en modelización de calidad del aire (en formato espacial y temporal), tomando como caso de estudio el Inventario Nacional de Emisiones de México 2008.

\section{MATERIALES Y MÉTODOS}

A partir del inventario anual de emisiones 2008 generado por la Secretaría del Medio Ambiente y Recursos Naturales (SEMARNAT), disponible en el subsistema del Inventario Nacional de Emisiones a la Atmósfera de México de entidades federativas y municipios (SINEA 2015), se obtienen las emisiones anuales por municipio de siete contaminantes (COV, $\mathrm{SO}_{2}, \mathrm{NH}_{3}, \mathrm{NOx}, \mathrm{CO}, \mathrm{PM}_{10}, \mathrm{PM}_{2.5}$ ). Las emisiones de este inventario están dadas por tres tipos de fuentes: fijas, móviles y de área, con sus respectivas categorías (64, 17 y 258 , respectivamente). A continuación se presenta la secuencia de acciones realizadas para la conversión del inventario, las cuales se ilustran en la figura 1: 


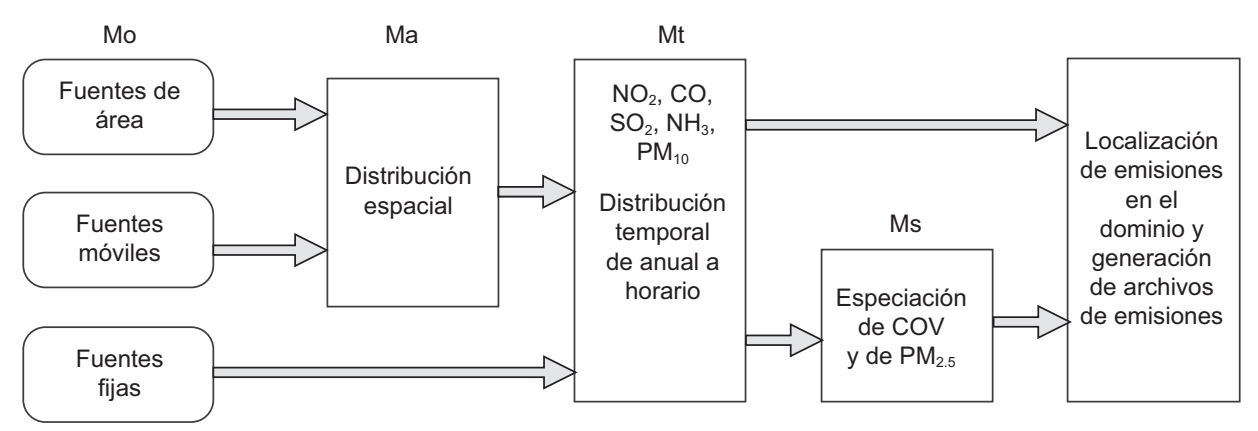

Fig. 1. Procedimiento empleado por el modelo para la conversión de emisiones anuales a emisiones para modelación. Mo: masa original del inventario; Ma: masa después de distribución espacial; Mt: masa después de distribución temporal; Ms: masa después de la especiación de compuestos orgánicos volátiles (COV) y partículas PM2.5. Los compuestos inorgánicos (e.g., $\left.\mathrm{SO}_{2}, \mathrm{CO}, \mathrm{NH}_{3}\right)$ no tienen especiación

1. Distribución espacial.

2. Distribución temporal.

3. Especiación química.

4. Agrupamiento en especies químicas para el mecanismo fotoquímico.

5. Herramientas de conversión.

\section{Distribución espacial}

La primera etapa consistió en generar una retícula que cubre la República Mexicana, cuyo centro se ubica en las coordenadas $24.06^{\circ} \mathrm{N}, 102.03^{\circ} \mathrm{O}$, y posee $353(\mathrm{E}-\mathrm{O})$ y $226(\mathrm{~S}-\mathrm{N})$ celdas cuadradas, cada una de aproximadamente $9 \mathrm{~km}$ por lado. La distribución espacial se realizó con una combinación de herramientas que incluyeron Microsoft Excel ${ }^{\circledR}$ y ArcGis ${ }^{\circledR}$ v.10.2. La información está referida a la proyección cartográfica cónica conforme de Lambert (CCL) y el Datum International Terrestrial Reference Frame 92 (ITRF92) época 1988.0 y a una escala 1:250 000. Para ubicar las emisiones en el área de estudio se utilizaron los datos vectoriales de uso de suelo, población, vialidades y carreteras, los cuales están en archivos de formato shape.

Las emisiones de área provenientes de fuentes antrópicas se distribuyeron proporcionalmente conforme a la población. Las emisiones relacionadas con las actividades agropecuarias emplearon el tipo de uso de suelo agrícola y las emisiones por incendios forestales se distribuyeron utilizando las categorías de bosque, selva, pastizal y matorral (vegetación). Para cada celda del inventario se utilizó la fracción de uso de suelo en la celda con referencia a la superficie de ese uso de suelo en el municipio, como se muestra en el cuadro I. Se utilizan los códigos de clasificación de fuente (SCC) que utiliza la Agencia de Protección Ambiental de Estados Unidos (EPA) para clasificar los diferentes tipos de actividades que generan emisiones (EPA 2018).

En el caso de las emisiones de fuentes móviles, se emplearon las carreteras y vialidades urbanas. Las categorías de fuentes vehiculares se presentan en el cuadro II. Para cada municipio y celda se obtuvo la fracción de carreteras y vialidades que las atraviesan, y se calculó el área de la vialidad correspondiente con el número de carriles. Las emisiones municipales se repartieron en cada celda a partir de la fracción obtenida del área de vialidad en la celda, entre el área de vialidad en el municipio.

Los datos del Instituto Nacional de Estadística y Geografía (INEGI) empleados para realizar la distribución espacial fueron los siguientes:

- Marco Geoestadístico Nacional 2010 v. 5.0A (INEGI 2011a)

- Conjunto de Datos Vectoriales de Uso del Suelo y Vegetación Serie V (2007-2010), escala 1:250 000 (INEGI 2013)

- Censo de Población y Vivienda 2010, Principales resultados por área geoestadística básica (AGEB) (INEGI 2011b)

- Conjunto de datos vectoriales de carreteras y vialidades urbanas edición 1.0 (INEGI 2011c)

En la figura 2 se presenta, a manera de ejemplo para el centro de México, la información de uso de suelo, vialidades, AGEB urbanas empleadas para realizar la distribución espacial de las fuentes de área y móviles, y las ubicaciones de las fuentes fijas.

En el caso de las fuentes fijas, se utilizó la ubicación de las 24133 chimeneas que incluyen los sectores de generación de energía, químico, alimentario, de papel y de extracción de petróleo y gas, como los 
CUADRO I. CÓDIGO DE CLASIFICACIÓN DE FUENTE (SCC, POR SUS SIGLAS EN INGLÉS), DESCRIPCIÓN Y COBERTURA DE SUELO USADA COMO VARIABLE DE DESAGREGACIÓN ESPACIAL

\begin{tabular}{|c|c|c|c|c|c|}
\hline SCC & Descripción & $\begin{array}{l}\text { Cobertura de } \\
\text { suelo }\end{array}$ & $\mathrm{SCC}$ & Descripción & $\begin{array}{l}\text { Cobertura de } \\
\text { suelo }\end{array}$ \\
\hline 2102004000 & Combustión & Población total & 2401990000 & Otra industria & $\mathbf{P}_{2}+2$ \\
\hline 2102007000 & Combustión industrial LPG & Población total & 2415000000 & Limpieza & Población urbana \\
\hline 2103006000 & Combustión industrial GN & Población urbana & 2420000055 & Lavado en seco & Población urbana \\
\hline 2103007000 & Combustión comercial diésel & Población urbana & 2425000000 & Imprenta & Población urbana \\
\hline 2104006000 & Combustión residencial NG & Población urbana & 2425010000 & Offset & Población urbana \\
\hline 2104007000 & Combustión residencial gas LP & Población urbana & 2425030000 & Rotograbado & Población urbana \\
\hline 2104008000 & Combustión residencial leña & Población rural & 2425040000 & Flexografía & Población urbana \\
\hline 2104011000 & Combustión residencial. queroseno & Población rural & 2461020000 & Asfaltado & Población total \\
\hline 2201070000 & Terminal de camiones & Población total & 2461850000 & Aplicación de pesticidas & Área agrícola \\
\hline 2222222222 & Cruces fronterizos & Población total & 2465000000 & Productos en aerosol & Población total \\
\hline 2260002000 & Maquinaria para construcción & Población total & 2465100000 & Productos uso personal & Población total \\
\hline 2267000000 & Combustión agrícola LPG & Área agrícola & 2465200000 & Productos domésticos & Población total \\
\hline \multirow[t]{2}{*}{2270005000} & Maquinaria & & & & \\
\hline & agríc & Área agrícola & 2465400000 & & Población urbana \\
\hline \multirow{2}{*}{$\begin{array}{l}2275000000 \\
2275050000\end{array}$} & Aviación & Población total & 2465600000 & Adhesivos y selladores & Población total \\
\hline & & & & Pesticidas comerciales y & \\
\hline \multirow{3}{*}{$\begin{array}{l}2280000000 \\
2285000000 \\
2285002010\end{array}$} & Embarc & & 2465900000 & $\begin{array}{l}\text { reside } \\
\text { Prodi }\end{array}$ & Población total \\
\hline & Locomotoras de arrastre & Población total & 2501000000 & Distribución gas LP & Población urbana \\
\hline & & & & Distribución de gasolina & \\
\hline \multirow{3}{*}{$\begin{array}{l}2302002000 \\
2302050000\end{array}$} & Locomotoras de patio & Población total & 2501060000 & estaciones & Población total \\
\hline & Asados al carbón & Población total & 2630030000 & $\begin{array}{l}\text { Plantas de tratamiento de } \\
\text { aguas }\end{array}$ & Población total \\
\hline & Pani & $P$ & 000 & $\begin{array}{l}\text { Combustión diesel para } \\
\text { agricultura }\end{array}$ & ícola \\
\hline 2311010000 & Const & total & 2801000002 & Labro & rícola \\
\hline 2401001000 & Recubrimientos & Población total & 2801500002 & Terminales c & Población urbana \\
\hline 2401005000 & Pintado automotriz & Población total & 2801500250 & Quemas agrícolas & Área agrícola \\
\hline 2401008000 & Señalización & Población total & 2801700000 & Aplicación de fertilizantes & Área agrícola \\
\hline 2401020000 & Solventes, recubrimiento & Población total & 2805000000 & Actividades ganaderas & Área agrícola \\
\hline 2401050000 & Fabricación de productos metálicos & Población total & 2805020000 & Emisiones de ganado & Área agrícola \\
\hline 2401055000 & Fabricación de maquinaria y equipo & Población total & 2810001000 & Incendios forestales & Área vegetación \\
\hline \multirow{3}{*}{$\begin{array}{l}2401065000 \\
2401080000 \\
2401100000\end{array}$} & $\begin{array}{l}\text { Uso de solventes para recubrimiento } \\
\text { industrial }\end{array}$ & Población total & 2810030000 & Incendios estructuras & Población total \\
\hline & $\begin{array}{l}\text { Recubrimiento en la fabricación de } \\
\text { equipo de transporte }\end{array}$ & Población total & 2850000010 & Hospitales & Población total \\
\hline & $\begin{array}{l}\text { Uso de solventes para recubrimiento } \\
\text { industrial }\end{array}$ & 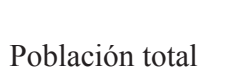 & & $\mathrm{H}_{3}$ uso reside & \\
\hline
\end{tabular}

CUADRO II. FUENTES VEHICULARES. CÓDIGO DE CLASIFICACIÓN DE FUENTE (SCC), COMBUSTIBLE Y DESCRIPCIÓN

\begin{tabular}{llllll}
\hline SCC & Combustible & Descripción & SCC & Combustible & Descripción \\
\hline 2230075330 & Diésel & Autobuses pesados (escolares y tránsito) & 2230071330 & Diésel & Vehículos pesados (HDDV) \\
2201020330 & Gasolina & Camiones ligeros (LDGT1) & 2230060330 & Diésel & Camionetas ligeras (LDDT) \\
2201040330 & Gasolina & Camiones ligeros (LDGT2) & 2230001330 & Diésel & Vehículos ligeros (LDDV) \\
2201070330 & Gasolina & Vehículos pesados (HDGV) & 2267000000 & GLP & Vehículos ligeros \\
2230072330 & Diésel & Vehículos pesados (HDDV) & 2268000000 & GNC & Vehículos ligeros \\
2230073330 & Diésel & Vehículos pesados (HDGV) & 2267001030 & GLP & Vehículos ligeros \\
2230074330 & Diésel & Vehículos pesados (HDGV) & 2268001030 & GNC & Vehículos ligeros \\
2201080330 & Gasolina & Motocicletas & 2268002066 & GNC & Equipos pesados \\
2201001330 & Gasolina & Vehículos ligeros (LDGV) & & & \\
\hline
\end{tabular}

(LDGV: light duty gasoline vehicle, LDGT1; light duty gasoline truck 1, LDGT2: light duty gasoline truck 2, HDGV: heavy duty gasoline vehicle, HDDV: heavy duty diesel vehicle, LDDT: light duty diesel truck, GLP: gas LP, GNC: gas natural comprimido) 


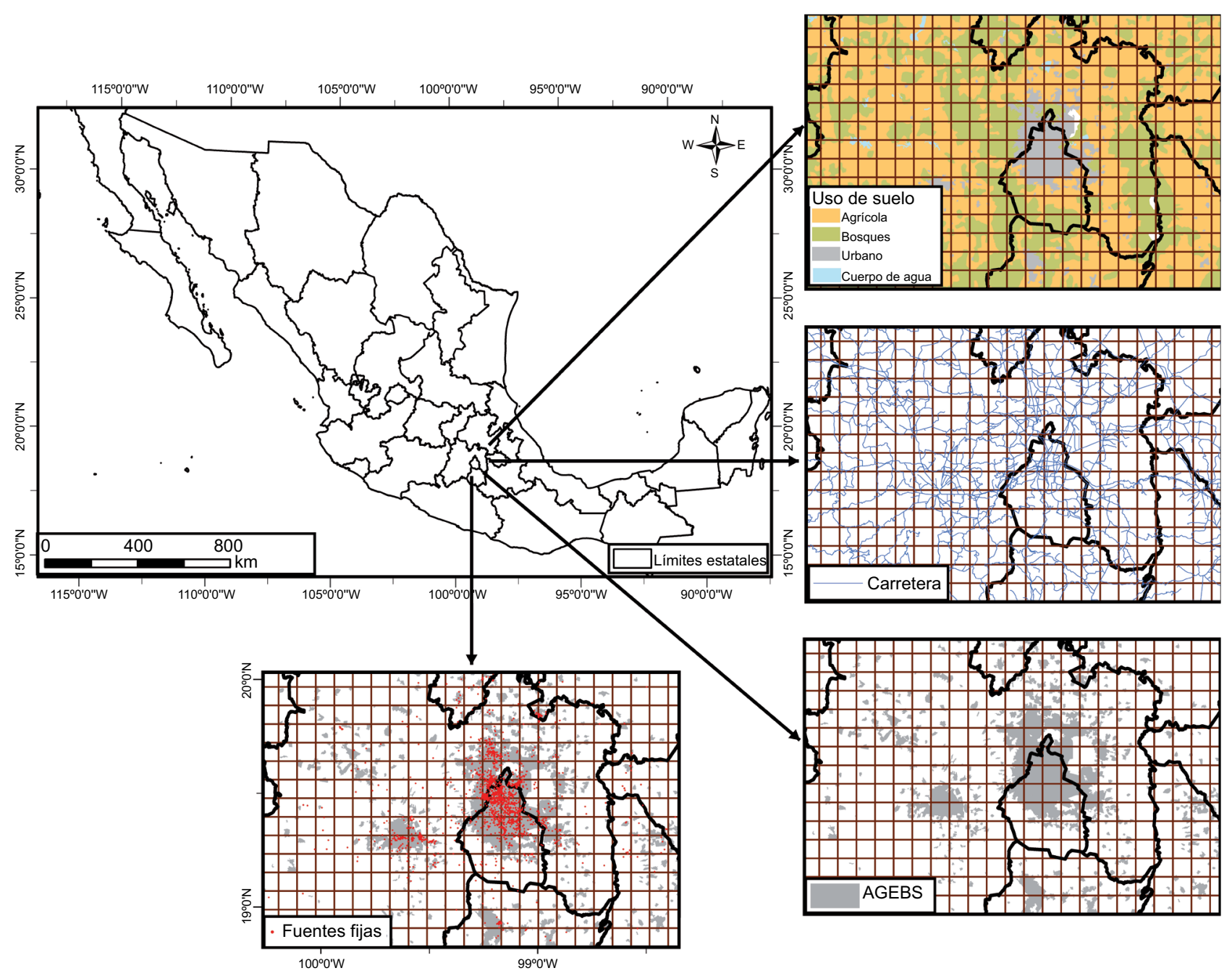

Fig. 2. Ejemplo de área de cobertura de la malla del inventario (superior izquierda) y las capas empleadas para la distribución espacial de las emisiones: uso de suelo (superior derecha), vialidades (centro derecha), áreas geostadísticas básicas (AGEBS) de población urbana (inferior derecha, en gris) y ubicaciones de fuentes puntuales (inferior centro)

más importantes (SINEA 2015). En las emisiones de fuentes fijas es importante considerar la altura de chimenea (Guevara et al. 2014); para calcularla se empleó información de las características (diámetro y altura) y de las condiciones de los gases de salida (temperatura, velocidad), así como las ecuaciones propuestas por Briggs (1984) para condiciones neutras como primera aproximación. Dependiendo de esta altura efectiva, las emisiones se ubican en la capa vertical correspondiente.

\section{Distribución temporal}

Para cada una de las categorías de emisión se identificó el SCC y a partir de éste se pudo determinar el perfil temporal (mensual, diario y horario). Estos perfiles provienen de información generada por la
EPA (2011a). El perfil temporal considera variaciones dependiendo del mes, día de la semana y hora del día. Para ello se emplean 18833 SCC, los cuales se relacionan con 980 perfiles temporales mensuales, 44 semanales y 188 horarios. La distribución temporal considera las tres zonas horarias (Centro, Pacífico y Noroeste) de la República Mexicana, vigentes en 2008.

\section{Especiación}

La categoría de COV considera la emisión de un conjunto de diferentes especies químicas, cuya proporción depende de la fuente y su proceso (e.g., combustión, recubrimientos, uso de solventes, etc.). Para la especiación se empleó el SCC de cada categoría de emisión (EPA 2011b). Esta información es sólo para partículas $\mathrm{PM}_{2.5}$ y COV de fase gas. En el 
caso de estos últimos, se tienen considerados 10487 códigos SCC, los cuales se relacionan con 393 perfiles de especiación. Esto se debe a que se pueden obtener diferentes números SCC que identifican a la combustión diésel en fuentes residenciales, agrícolas, comerciales y puntuales. A todos ellos les corresponde el mismo perfil de especies químicas.

\section{Agrupamiento en especies químicas para el me- canismo fotoquímico}

Las especies químicas individuales obtenidas en el paso anterior no se emplean directamente en el cálculo de la fotoquímica, debido a que los mecanismos no consideran especies individuales porque se requiere de un uso masivo de recursos de cómputo, de manera que estas substancias se agrupan dependiendo del mecanismo químico.

En esta etapa se empleó la información elaborada por Carter (2015) para agrupar los compuestos químicos en categorías de los mecanismos químicos RADM2 (Stockwell et al. 1990), RACM (Goliff et al. 2013) y SAPRC99 (Carter 2000).

Para cada categoría del mecanismo químico se suman las emisiones de las especies químicas que incluye dicha categoría, mediante un factor de agregación (fa) (Middleton et al. 1990). Adicionalmente, en este paso, se convierten las emisiones de masa a mol por medio del peso molecular de la categoría.

\section{Herramientas de conversión}

Las herramientas de conversión son un conjunto de programas escritos en Fortran y otros programas auxiliares escritos en comandos de C-shell (csh), con el objeto de automatizar el proceso de conversión del inventario. Estas herramientas se distribuyen en diez etapas que se describen en el cuadro III.

Se desarrolló un programa auxiliar en csh para la generación del inventario para fechas específicas de 2008, en el cual se indica mes, día inicial y final del periodo para obtener el inventario.

El inventario de emisiones se guarda en un archivo digital con formato NetCDF (Rew y Davis 1990). El modelo WRF-chem (Grell et al. 2005) requiere la estructura del archivo y los nombres de las variables para emisiones antrópicas.

Los archivos de salida contienen 38 categorías para el mecanismo RADM2, 37 para RACM y 55 para SAPRC99, y tienen hasta cinco capas en la vertical $(17.5,35,56,70$ y $142 \mathrm{~m})$. Los archivos son generados por día (un archivo contiene $24 \mathrm{~h}$ ).

De cada categoría de emisión del INEM 2008 se obtuvieron las especies químicas que luego se reagruparon en las categorías de los mecanismos químicos. En la figura 3 se presenta la distribución de los compuestos orgánicos en las principales categorías de cada mecanismo químico utilizado (RADM2, RACM2 y SAPRC99).

\section{CUADRO III. ETAPAS PARA LA CONVERSIÓN DEL INVENTARIO}

\begin{tabular}{cl}
\hline Etapa & Descripción de programas (Fortran) \\
\hline 1 & Suma de la población rural y urbana en cada celda \\
\hline 2 & $\begin{array}{l}\text { Distribución espacial de las emisiones de fuentes de área con base en la distribución espacial de la población y las clases de } \\
\text { uso de suelo (agrícola y bosque) } \\
\mathrm{CO}, \mathrm{NOx}, \mathrm{SO}_{2}, \mathrm{COV}, \mathrm{NH}_{3}, \mathrm{PM}_{10}, \mathrm{PM}_{2.5}\end{array}$ \\
\hline 3 & Obtención de la fracción de vialidad. Superficie de vialidad en la celda sobre la superficie de vialidad en el municipio \\
\hline 4 & Distribución temporal de las emisiones de área generadas en la etapa 2 \\
\hline 5 & $\begin{array}{l}\text { Distribución espacial de las emisiones de fuentes móviles }\left(\mathrm{CO}, \mathrm{NOx}, \mathrm{SO}_{2}, \mathrm{COV}, \mathrm{NH}_{3}, \mathrm{PM}_{10}, \mathrm{PM}_{2.5}\right) \text { con la fracción de carretera } \\
\text { y vialidad de la etapa } 3\end{array}$ \\
\hline 6 & Distribución temporal de las emisiones de fuentes móviles generadas en la etapa 5 \\
\hline 7 & Distribución temporal, identificación de la altura de emisión y ubicación en la malla de las emisiones de fuentes fijas \\
\hline 8 & $\begin{array}{l}\text { Distribución en especies químicas de las emisiones de compuestos orgánicos volátiles y agrupamiento en las clases del mecanismo } \\
\text { químico seleccionado. Emplea las salidas de las etapas } 4,6 \text { y } 7\end{array}$ \\
\hline 9 & Distribución de las partículas PM 2.5 en las diferentes fracciones orgánicas e inorgánicas. Emplea las salidas de las etapas 4,6 y 7 \\
\hline 10 & Generación del archivo de emisiones \\
\hline
\end{tabular}



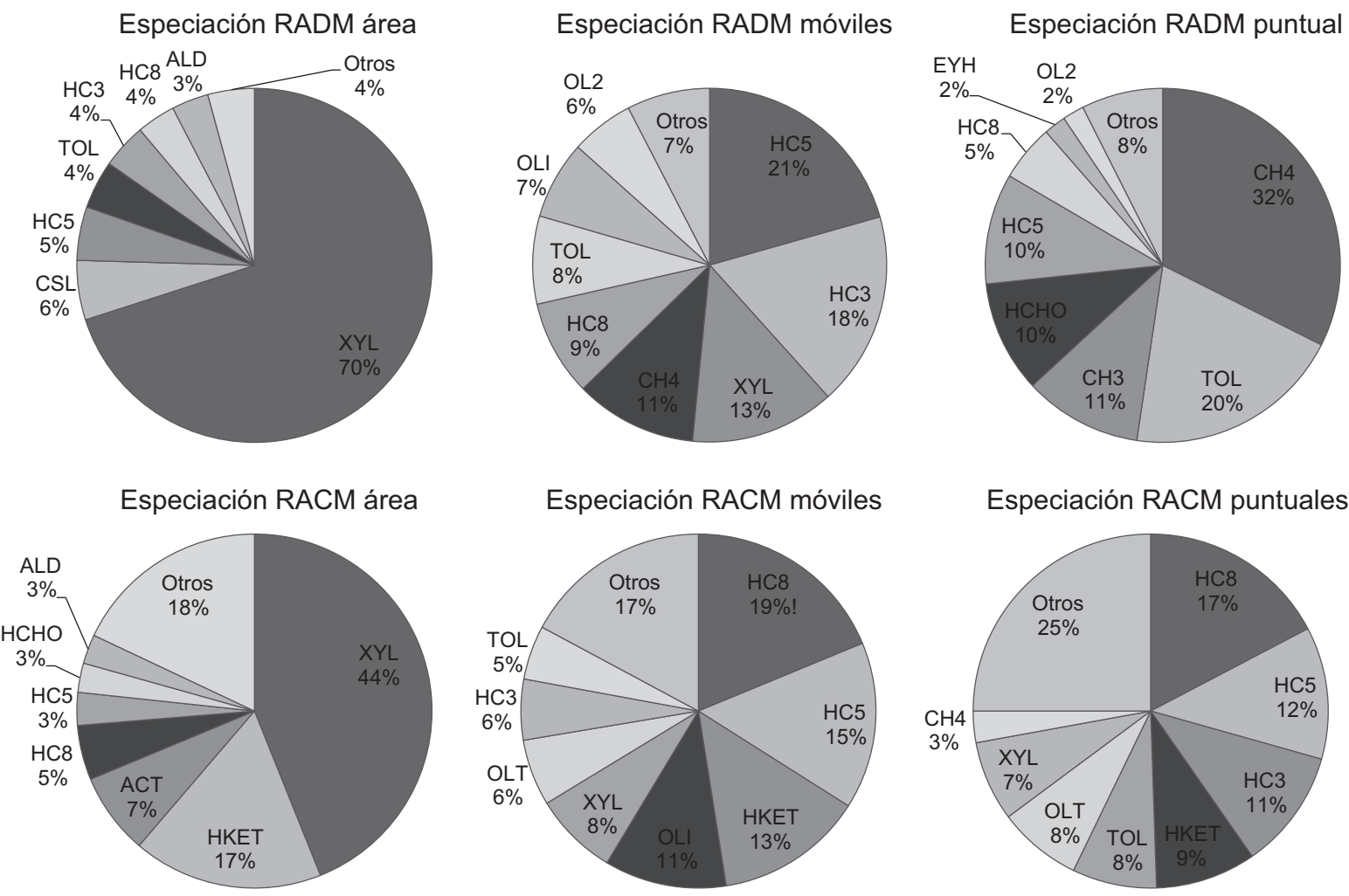

\section{Especiación SAPRC99 área}

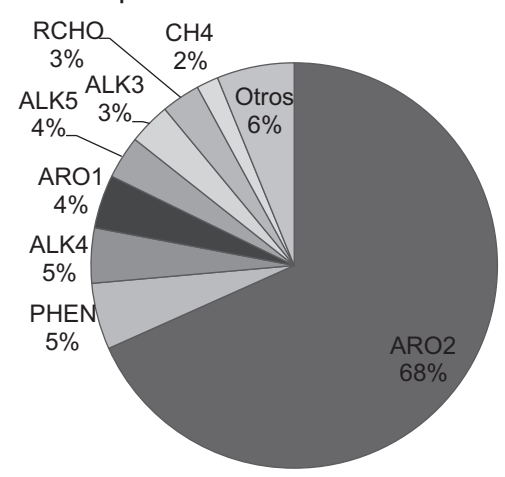

Especiación SAPRC99 móviles

Especiación SAPRC99 puntuales
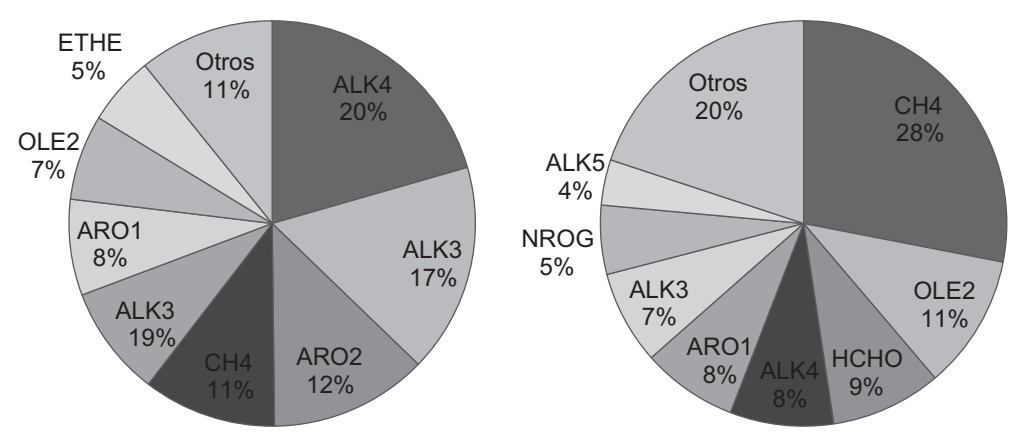

Fig. 3. Especiación del inventario nacional de emisiones para compuestos orgánicos volátiles $(\mathrm{COV})$ por tipo de emisión y para cada uno de los mecanismos fotoquímicos: Regional Acid Deposition Model (RADM), Regional Atmospheric Chemistry Mechanism (RACM) y Statewide Air Pollution Research Center Mechanism (SAPRC99). $\mathrm{k}_{\mathrm{oh}}$ : constante de reactividad con radical OH; ACT: acetona; ALD: aldehídos; ALK3: alcanos y no aromáticos con koH de 0.5 a 2.5; ALK4: alcanos y no aromáticos con $\mathrm{k}_{\mathrm{OH}}$ de 2.5 a 5; ALK5: alcanos y no aromáticos con $\mathrm{k}_{\mathrm{OH}}$ de 0.5 a 2.5; ARO1: aromáticos con $\mathrm{k}_{\mathrm{OH}}<2$; $\mathrm{ARO} 2$, aromáticos con $\mathrm{k}_{\mathrm{OH}}>$ 2; CH4: metano; CSL: cresol; ETH: etano; ETHE: etileno; $\mathrm{HC}_{3}$ : propano; $\mathrm{HC}_{5}$ : alcanos $\left(\mathrm{k}_{\mathrm{OH}}\right.$ de 0.5 a 1.0); $\mathrm{HC}_{8}$ : alcanos $\mathrm{HC} 3, \mathrm{HC} 5, \mathrm{HC} 8$ ( $\mathrm{k}_{\mathrm{OH}}$ de 1 a 2); $\mathrm{HCOH}$ : formaldehído; HKET: hidroxicetona; NROG: no reactivos; OLE2: alquenos ( $\left.\mathrm{k}_{\mathrm{OH}}>7\right)$; OLI: alquenos internos; OLT: alquenos terminales; PHE: fenol; TOL: tolueno; XYL: xilenos

Se observa que los compuestos aromáticos, como el xileno (XYL y ARO2), constituyen la categoría principal en las fuentes de área. En el caso de fuentes móviles la categoría principal está formada por los alcanos (HC3, HC5, HC8, ALK4 y ALK3), en las fuentes fijas por el metano $\left(\mathrm{CH}_{4}\right)$, y en el caso de
RADM2 y SAPRC99 y para el mecanismo RACM, por los alcanos. Otros compuestos como la hidroxi acetona (HKET), acetona (ACT), tolueno (TOL), aldehídos (ALD), alquenos terminales (OLT), alquenos intermedios (OLI) y formaldehído (HCHO) integran categorías con emisiones considerables. 

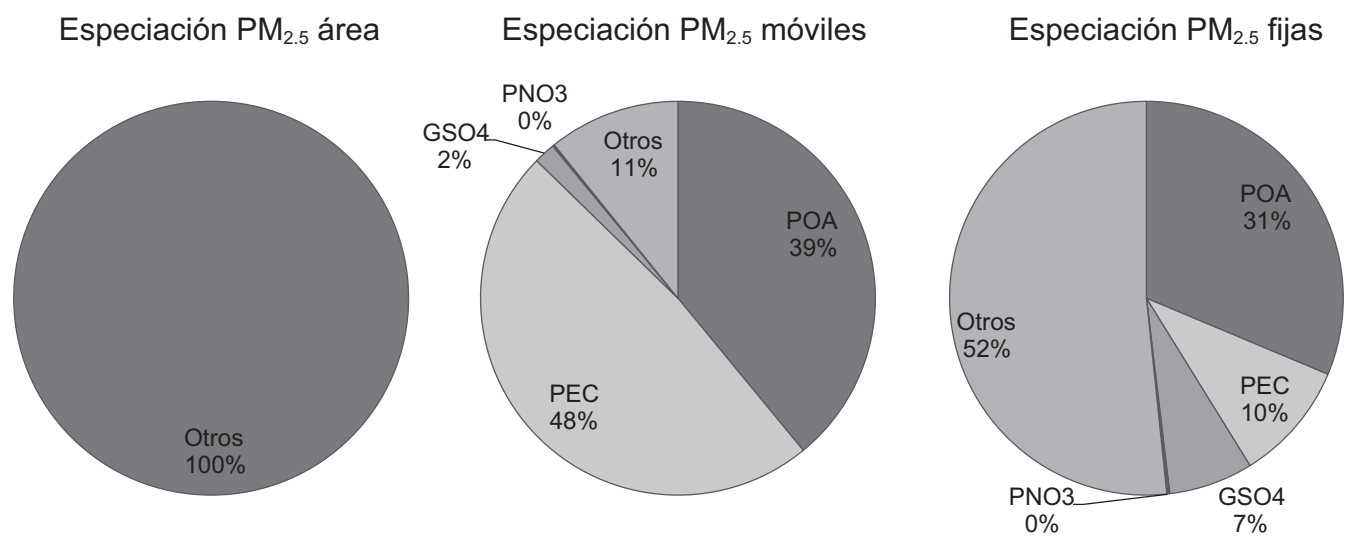

Fig. 4. Especiación del inventario de emisiones de $\mathrm{PM}_{2.5}$ por tipo de emisión. (PNO3: nitratos; POA: aerosol orgánico; PEC: partículas de carbono elemental; $\mathrm{GSO}_{4}$ : sulfatos)

En el caso de partículas menores a $2.5 \mu \mathrm{m}$, los resultados de la aplicación del modelo de emisiones al INEM 2008 se agruparon en cinco categorías (POA, aerosoles primarios orgánicos; PEC, partículas de carbono elemental; GSO4, sulfatos; PNO3, nitratos, y OTHER, otros) que se muestran en la figura 4. En las fuentes de área sólo se tiene 22 categorías que emiten partículas y todas ellas corresponden al tipo OTHER (en las que se incluye el material geológico). En las fuentes móviles, la categoría principal son las PEC, seguidas por los POA. En las fuentes fijas, los OTHER corresponden a más de la mitad de las emisiones, seguidos por las categorías POA, PEC y GSO4. Estas últimos son las que se encuentran en menor proporción.

Para validar el modelo se comparó la masa de cada compuesto del inventario original con la masa obtenida de ese mismo compuesto después de cada paso de la conversión. Así, la masa total después de la distribución espacial (Ma) debe ser igual a la masa del inventario original (Mo), y la masa obtenida después de la distribución temporal (Mt) debe ser igual a la Mo modificada por el ajuste. Hay que tomar en cuenta que la emisión después de la especiación temporal pasa de $\mathrm{Mg} / \mathrm{año}$ a $\mathrm{g}$ /hora, por lo que se suman las emisiones horarias para obtener la emisión diaria; posteriormente, se multiplica por el número de días al año para obtener la emisión anual (Mt) y ésta se compara con la emisión anual original (Mo); finalmente, después de la conversión a especies químicas y agrupamiento en categorías de emisión, la masa obtenida de las categorías (Ms) debería ser igual a la masa original del inventario Mo (Fig. 1).

\section{RESULTADOS}

Mediante el procedimiento descrito con anterioridad se pueden generar archivos diarios con las emisiones de México que pueden utilizarse en la modelización de calidad del aire. Los archivos contienen las emisiones horarias de las especies del mecanismo químico seleccionado (RADM2, RACM o SAPRC99). En las figuras 5 y $\mathbf{6}$ se muestra la distribución espacial del monóxido de carbono (CO), aldehído (ALD), propano (HC3), óxido de nitrógeno (NO) y dióxido de azufre $\left(\mathrm{SO}_{2}\right)$.

En la figura 7 se presenta la distribución temporal de las emisiones durante una semana para $\mathrm{CO}$ en el centro de la Ciudad de México. La emisión varía de acuerdo con la hora y el día, teniendo en cuenta que en los dos primeros días se presenta el fin de semana, que inicia el viernes a las 18:00 horas. También se observan diferencias entre cada día de la semana.

\section{Validación}

La validación del método presentado se realizó mediante la comparación de resultados en cada uno de los pasos para la conversión del inventario original. En el cuadro IV se presenta la comparación entre el inventario original y la etapa de distribución espacial y distribución temporal. Para la distribución temporal hay actividades que varían con la estación, por lo que el resultado obtenido al multiplicar la emisión de un día para obtener la anual puede presentar diferencias importantes. Con el objeto de validar el procedimiento utilizado para la distribución temporal, se emplearon perfiles que dieran una distribución homogénea durante el año para cada uno de los SCC; 


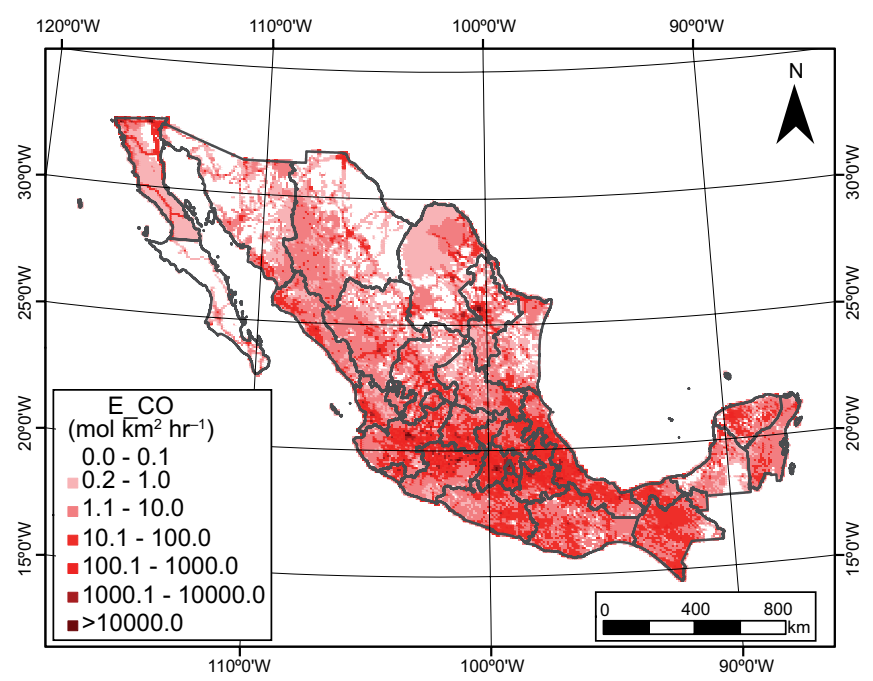

Fig. 5. Distribución espacial de emisiones de monóxido de carbono para el 10 de abril a las 18:00 horas
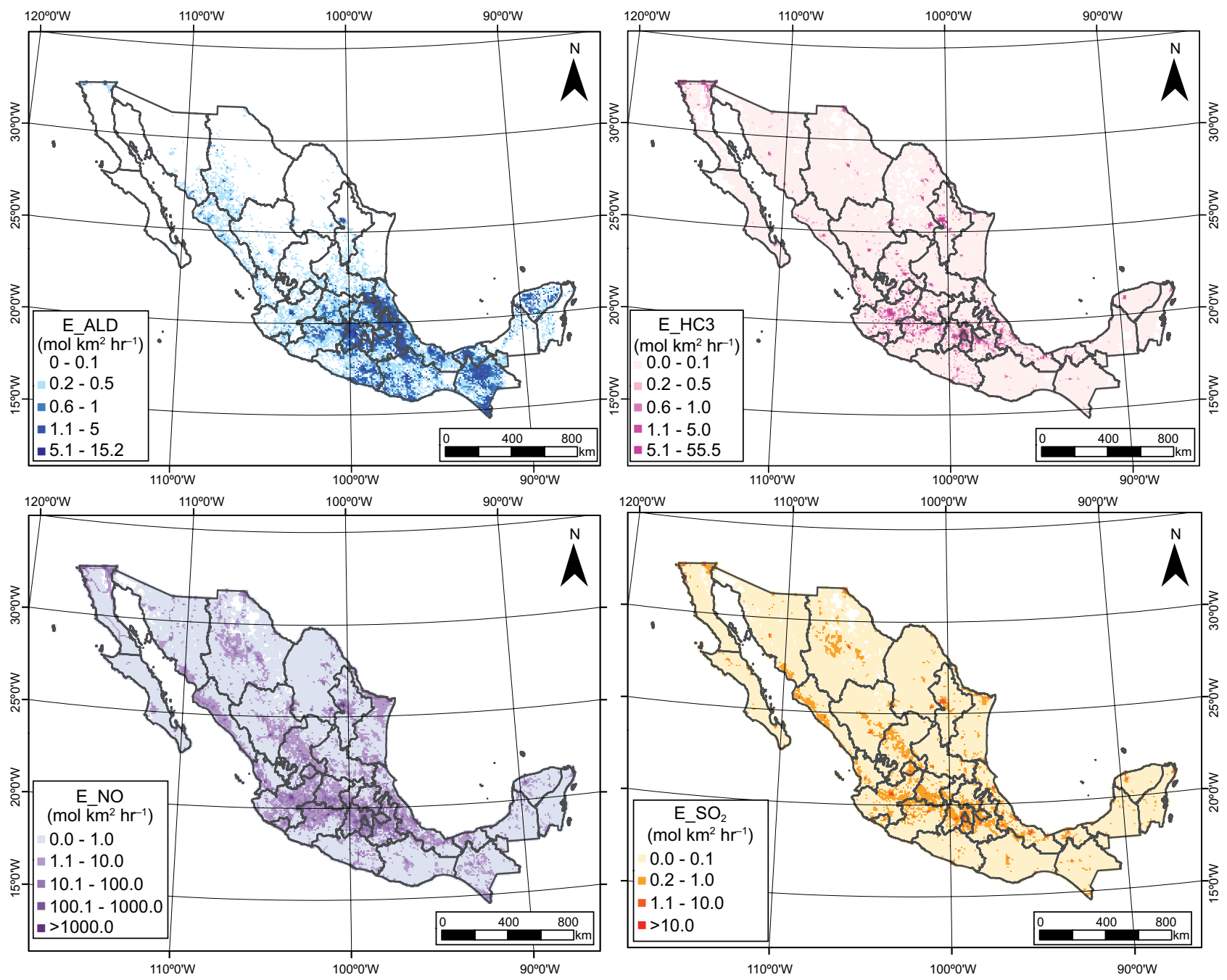

Fig. 6. Distribución espacial de emisiones de aldehídos (superior izquierda), propano (superior der.), óxido de nitrógeno (inferior derecha) y dióxido de azufre del 10 de abril a las 18:00 horas 


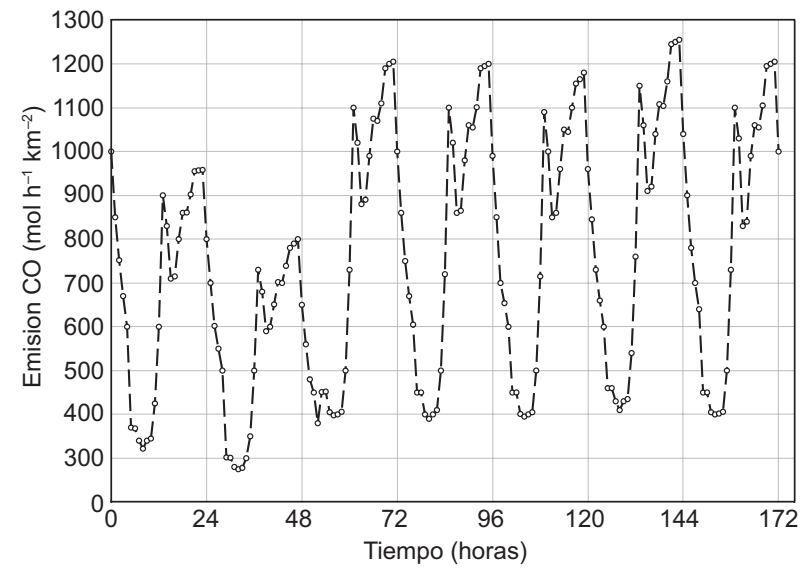

Fig. 7. Distribución horaria de las emisiones de monóxido de carbono para una semana de abril de 2008, para un sitio en la Ciudad de México. Los valores en el eje horizontal son horas e inicia el viernes a las 18:00 horas, tiempo local

así, la emisión anual se distribuyó en 1/12 para cada mes, 1/7 para cada día de la semana y 1/24 para cada hora del día. Los resultados del empleo de esta distribución se incluyen en el cuadro IV.

Se aprecia que en la etapa de distribución espacial la diferencia entre resultados es de hasta $-2.5 \%$ en las emisiones de $\mathrm{NH}_{3}$ por fuentes de área. Esto se debe a que hay emisiones de $\mathrm{NH}_{3}$ generadas por actividades ganaderas y de aplicación de fertilizantes que no se encuentran en zonas agrícolas, por lo que no se asignan.

Tampoco se aprecia una diferencia significativa en las fuentes móviles ni en la etapa de distribución temporal en relación con la masa de emisión obtenida de la distribución espacial. En el caso de fuentes puntuales, la diferencia entre los resultados de la distribución temporal y las emisiones originales es de hasta el $1.2 \%$.

En el tema de la distribución de los COV en especies químicas y su agrupación en categorías del mecanismo químico, se tiene la emisión antes y después de este proceso, como se muestra en el cuadro $\mathbf{V}$. Se puede apreciar que existe una reducción entre la masa original y la masa que se obtiene después del proceso de especiación-agrupación.

La diferencia entre el valor inicial y el obtenido después de procesar en especies se debe, principalmente, a que un gramo de un compuesto químico puede contribuir con una fracción a varias categorías del mecanismo, dependiendo de su reactividad (Middleton et al. 1990), y una de esas categorías es la

CUADRO IV. EMISIONES POR TIPO DE FUENTE Y EN CADAETAPA DE LA CONVERSIÓN (Gg/año). LA DIFERENCIA CORRESPONDE AL VALOR DE LA MASA OBTENIDO DESPUÉS DEL PROCESO CON REFERENCIA AL VALOR ORIGINAL

\begin{tabular}{|c|c|c|c|c|c|c|}
\hline Fuente & Compuesto & Original & $\begin{array}{l}\text { Distribución } \\
\text { espacial }\end{array}$ & $\begin{array}{c}\% \\
\text { diferencia }\end{array}$ & $\begin{array}{l}\text { Distribución } \\
\text { temporal }\end{array}$ & $\begin{array}{c}\% \\
\text { diferencia }\end{array}$ \\
\hline \multirow[t]{7}{*}{ Área } & $\mathrm{CO}$ & 2796 & 2789 & -0.3 & 2789 & -0.3 \\
\hline & $\mathrm{NH}_{3}$ & 769.0 & 749.6 & -2.5 & 749.5 & -2.5 \\
\hline & NOx & 654.0 & 650.9 & -0.5 & 650.8 & -0.5 \\
\hline & $\mathrm{COV}$ & 3143 & 3139 & -0.1 & 3132 & -0.3 \\
\hline & $\mathrm{PM}_{10}$ & 570.0 & 566.5 & -0.6 & 566.5 & -0.6 \\
\hline & $\mathrm{PM}_{2.5}$ & 412.3 & 410.8 & -0.4 & 410.5 & -0.4 \\
\hline & $\mathrm{SO}_{2}$ & 42.90 & 42.81 & -0.2 & 42.80 & -0.2 \\
\hline \multirow[t]{7}{*}{ Móviles } & $\mathrm{CO}$ & 29611 & 29599 & 0.0 & 29597 & 0.0 \\
\hline & $\mathrm{NH}_{3}$ & 62.22 & 62.19 & 0.0 & 62.19 & 0.0 \\
\hline & NOx & 2005 & 2004 & -0.1 & 2004 & -0.1 \\
\hline & $\mathrm{COV}$ & 2599 & 2598 & 0.0 & 2597 & -0.1 \\
\hline & $\mathrm{PM}_{10}$ & 17.43 & 17.42 & -0.1 & 17.42 & -0.1 \\
\hline & $\mathrm{PM}_{2.5}$ & 12.53 & 12.52 & -0.1 & 12.51 & -0.2 \\
\hline & $\mathrm{SO}_{2}$ & 26.60 & 26.59 & 0.0 & 26.59 & 0.0 \\
\hline \multirow[t]{7}{*}{ Puntual } & $\mathrm{CO}$ & 142.0 & - & - & 140.7 & -0.9 \\
\hline & $\mathrm{NH}_{3}$ & 10.76 & - & - & 10.69 & -0.7 \\
\hline & NOx & 464.0 & - & - & 458.5 & -1.2 \\
\hline & $\mathrm{COV}$ & 68.22 & - & - & 67.69 & -0.8 \\
\hline & $\mathrm{PM}_{10}$ & 123.0 & - & - & 123.0 & 0.0 \\
\hline & $\mathrm{PM}_{2.5}$ & 93.00 & - & - & 93.49 & 0.5 \\
\hline & $\mathrm{SO}_{2}$ & 1407 & - & - & 1407 & 0.0 \\
\hline
\end{tabular}


CUADRO V. COMPARACIÓN ENTRE LA MASA DE LAS EMISIONES ORIGINALES DE LOS COMPUESTOS ORGÁNICOS VOLÁTILES (COV) PARA CADA MECANISMO Y LAS OBTENIDAS DESPUÉS DEL PROCESO DE ESPECIACIÓN-AGRUPACIÓN

\begin{tabular}{llccc}
\hline & & Móviles & Fijas & Área \\
\hline Mecanismo & Original (mg/día) & 8037 & 185 & 14489 \\
\hline RADM2 & Especiación (mg/día) & 6634 & 158 & 13584 \\
& \% diferencia & -17.4 & -14.6 & -6.2 \\
\hline \multirow{2}{*}{ RACM2 } & Especiación (mg/día) & 6447 & 175 & 1104 \\
& \% diferencia & -19.8 & -5.6 & -7.0 \\
\hline \multirow{2}{*}{ SAPRC99 } & Especiación (mg/día) & 6963 & 183 & 13927 \\
& \% diferencia & -13.4 & -1.3 & -3.9 \\
\hline
\end{tabular}

de compuestos orgánicos no reactivos. Éstos llegan a constituir hasta el $92 \%$ de la masa de una especie química, por lo que el total de la masa de las categorías no llega a sumar el total inicial, puesto que no todos los compuestos participan en la química atmosférica.

En el caso de la especiación de $\mathrm{PM}_{2.5}$ no se observa una diferencia apreciable entre la emisión original y la distribución en especies de partículas (cuadro VI).

CUADRO VI. COMPARACIÓN ENTRE LA MASA DE LAS EMISIONES ORIGINALES DE $\mathrm{PM}_{2.5}$ Y LAS OBTENIDAS DESPUÉS DEL PROCESO DE ESPECIACIÓN

\begin{tabular}{llcrc}
\hline & & Móviles & Fijas & Área \\
\hline $\mathrm{Mg} /$ día & Original & 33.72 & 251.60 & 1110 \\
\hline $\mathrm{PM}_{2.5}$ & Especiación & 33.68 & 251.63 & \multicolumn{1}{c}{1105} \\
& \% diferencia & 0.00 & 0.00 & 0.00 \\
\hline
\end{tabular}

Los valores obtenidos de diferencias entre el inventario original y el modelo de emisiones aquí expuesto, demuestran la idoneidad de este último para estimar la distribución espacial, temporal y de especiación.

\section{DISCUSIÓN}

El modelo que aquí se presenta es una primera aproximación a la conversión del inventario de emisiones 2008 de México a un inventario útil para la modelización con resolución de $9 \times 9 \mathrm{~km}$, a diferencia de otros que sólo consideran la zona urbana del Valle de México y alrededores. La validación del procedimiento muestra que el modelo de emisiones posee una diferencia menor del $2.5 \%$ para los compuestos inorgánicos y del $20 \%$ para los orgánicos.

La diferencia entre el valor inicial y el obtenido de los COV después de procesar en especies se debe, principalmente, a que un gramo de un compuesto químico puede contribuir a la categoría de compuestos orgánicos no reactivos, los cuales no participan en la química atmosférica; por ello, el total de la masa de las categorías no corresponde a la masa inicial de los COV. Es importante destacar este resultado, ya que debido a la manera en que se asigna la masa de las especies químicas en las categorías de los mecanismos de reacción, los COV de los inventarios no conservan la totalidad de su masa en los inventarios para modelización. Para el ejercicio aquí presentado se tiene una diferencia de hasta un $20 \%$ en masa. No se observaron diferencias en la especiación de las partículas $\mathrm{PM}_{2.5}$.

La información utilizada para la desagregación temporal y la especiación en este modelo de emisiones provienen de referencias extranjeras, por lo que es posible que los resultados de este modelo contengan errores sistemáticos. Lo anterior induciría a que un modelo de calidad del aire proporcione concentraciones diferentes a las observadas. La optimización del modelo de emisiones para que se generen concentraciones similares a las medidas es ámbito de otra investigación, fuera del alcance de este trabajo, que es importante realizar en un futuro próximo.

Dentro de los puntos a mejorar en este modelo de emisiones se encuentran los siguientes: continuar el trabajo sobre el mapeo de categorías de emisión en México, con los SCC; mejorar la distribución de emisiones mediante la inclusión de la ubicación de aeropuertos, estaciones de ferrocarril, centrales 
de autobuses y puertos marítimos, y actualizar los perfiles temporales para que sean representativos de la región de estudio. En cuanto a las fuentes móviles, la distribución de las emisiones se podría mejorar si se conoce el flujo vehicular horario, con lo cual se podrían asignar mejor las emisiones, tanto espacial como temporalmente. La aproximación realizada para distribuir las emisiones en las áreas de vialidad puede mostrar inconsistencias, como las que se observan entre Baja California y Baja California Sur, donde una carretera posee una emisión mayor que su continuación hacia el sur.

En la especiación se requieren estudios y mediciones de los sectores más importantes de emisiones de los COV para que reflejen la composición de las fuentes de la región. En cuanto a las fuentes puntuales, la altura efectiva de chimenea se debería calcular con la información meteorológica, con el objeto de incluir los valores correspondientes a cada hora.

Aunque el inventario de emisiones considera las fuentes biogénicas, este modelo no las consideró, ya que el modelo de calidad del aire WRF-chem puede generarlas a partir del uso de suelo y las variables meteorológicas.

Una vez calibrado el modelo de emisiones, se puede utilizar para modelar la calidad del aire y con ello evaluar las políticas de control, ya que tiene la facilidad de modificar fuentes específicas de emisiones para observar su efecto en la calidad del aire. Si bien el inventario de emisiones es de 2008, sus resultados pueden emplearse para estudios de calidad del aire de eventos pasados; adicionalmente, el procedimiento es útil para aplicarse a las nuevas versiones del inventario de emisiones.

\section{CONCLUSIÓN}

Para el control de la contaminación ambiental y evaluación del beneficio de aplicar medidas de control, el uso de modelos de calidad del aire es fundamental. A partir de este trabajo se presenta un modelo de emisiones que describe la metodología y programas útiles para convertir y usar el inventario anual de emisiones 2008 en las modelizaciones de calidad del aire.

Se empleó la información sobre población, vialidades y uso de suelo de dicho inventario mediante un sistema de información geográfico para distribuir las emisiones espacialmente; asimismo, se utilizó el código de clasificación de fuentes para identificar los perfiles horarios y la especiación, tanto de emisiones gaseosas, como de partículas.
El número y categorías de emisión son los requeridos para correr tres diferentes mecanismos químicos: RADM, RACM y SAPRC99. Se encontró que, como resultado del proceso de conversión, la masa de los COV es $20 \%$ menor que la inicial, debido a que existe una categoría de compuestos orgánicos no reactivos que no participa en la química atmosférica. En los productos del modelo se incluye la especiación de las partículas $\mathrm{PM}_{2.5}$, que contienen a las PEC. A partir de este trabajo se podrán realizar modificaciones al inventario, tanto para hacer estudios de sensibilidad, como para evaluar el impacto de medidas de control de emisiones.

El producto final es un archivo con las emisiones en una malla de $353(\mathrm{O}-\mathrm{E}) \times 226(\mathrm{~S}-\mathrm{N}) \times 6$ (verticales) en celdas de $9 \times 9 \mathrm{~km}$, para las emisiones de los gases inorgánicos $\mathrm{CO}, \mathrm{NO}, \mathrm{NO}_{2}, \mathrm{NH}_{3}$, $\mathrm{SO}_{2}$; partículas $\left(\mathrm{PM}_{10}\right.$ y $\left.\mathrm{PM}_{2.5}\right)$, y, en el caso del mecanismo RADM 30, categorías de compuestos orgánicos.

\section{AGRADECIMIENTOS}

Los autores agradecen a la Secretaria de Ciencia y Tecnología de la Ciudad de México por el financiamiento parcial de este trabajo a través del proyecto SECITI/055/2016, así como el apoyo de la Lic. Brenda Álvarez Carreño en la edición del texto.

\section{REFERENCIAS}

Almanza V.H., Molina L.T., Li G., Fast J. y Sosa G. (2014). Impact of external industrial sources on the regional and local $\mathrm{SO}_{2}$ and $\mathrm{O}_{3}$ levels of the Mexico megacity. Atmos. Chem. Phys. 14 (16) 8483-8499.

DOI: $10.5194 / \mathrm{acp}-14-8483-2014$

Briggs, G.A. (1984). Plume rise and buoyancy effects. En: Atmospheric science and power (Randerson D., Ed.). US Department of Energy, 327-366 [en línea]. https:/ntrl.ntis.gov/NTRL/dashboard/searchResults/ titleDetail/DE84005177.xhtml

Carter W.P.L. (2000). Documentation of the SAPRC-99 chemical mechanism for VOC reactivity assessment. California Air Resources Board, Research Division, EU [en línea]. http://www.engr.ucr.edu/ carter/pubs/ s99doc.pdf 7/12/2017

Carter W.P.L. (2015). Development of a database for chemical mechanism assignments for volatile organic emissions. J. Air Waste Manage. Assoc. 65 (10) 1171-1184.

DOI: $10.1080 / 10962247.2015 .1013646$ 
Emmons L.K., Apel E.C., Lamarque J.F., Hess P.G., Avery M., Blake D., Brune W., Campos T., Crawford J., DeCarlo P.F., Hall S., Heikes B., Holloway J., Jimenez J.L., Knapp D.J., Kok G., Mena-Carrasco M., Olson J., O’Sullivan D., Sachse G., Walega J., Weibring P., Weinheimer A.y Wiedinmyer C. (2010). Impact of Mexico City emissions on regional air quality from MOZART-4 simulations. Atmos. Chem. Phys. 10 (13) 6195-6212.

DOI: $10.5194 /$ acp-10-6195-2010

EPA (2011a). Emissions modeling temporal allocation (ge_dat_for_2011v3_temporal.zip). United States Environmental Protection Agency [en línea]. ftp:// ftp.epa.gov/EmisInventory/2011v6/v3platform/ancillary_data/ 10/10/2018

EPA (2011b). Source category code/speciation profile (ge_dat_for_2011v3_speciation.zip). United States Environmental Protection Agency [en línea]. ftp:// ftp.epa.gov/EmisInventory/2011v6/v3platform/ancillary_data/ 10/10/2018

EPA (2018). Source classification codes (SCCs). United States Environmental Protection Agency [en línea]. https://ofmpub.epa.gov/sccwebservices/sccsearch/ $10 / 10 / 2018$

García-Escalante J.S., García-Reynoso J.A., JazcilevichDiamant A. y Ruiz-Suárez L.G. (2014). The influence of the Tula, Hidalgo complex on the air quality of the Mexico City Metropolitan Area. Atmósfera 27 (2) 215 225. DOI: 10.1016/S0187-6236(14)71111-7

García-Reynoso J.A. (2002). Evaluacion de escenarios utilizando el modelo regional de calidad del aire multiscale climate chemistry model. Tesis de doctorado, Universidad Nacional Autónoma de México, 114 pp.

García-Reynoso J.A., Jazcilevich A., Ruiz-Suárez L.G., Torres-Jardón R., Suárez Lastra M. y Reséndiz Juárez N.A. (2009). Ozone weekend effect analysis in Mexico City. Atmósfera 22 (3) 281-297.

Goldstone M.E. (2013). Review of evidence on health aspects of air pollution - REVIHAAP project. Technical report. World Health Organization, regional office for Europe. Copenhague, Dinamarca [en línea]. http://www.euro.who.int/_data/assets/ pdf_file/0004/193108/REVIHAAP-Final-technicalreport-final-version.pdf?ua $=1$ 7/12/2017

Goliff W.S., Stockwell W.R. y Lawson C.V. (2013). The regional atmospheric chemistry mechanism, v. 2. Atmos. Environ. 68, 174-185.

DOI: $10.1016 /$ j.atmosenv.2012.11.038

Grell G.A., Peckham S.E., Schmitz R., McKeen S.A., Frost G., Skamarock W.C. y Eder B. (2005). Fully coupled online chemistry within the WRF model. Atmos. Environ. 39 (37) 6957-6975.

DOI: $10.1016 /$ j.atmosenv.2005.04.027
Guevara M., Soret A., Arévalo G., Martínez F. y Baldasano J. (2014). Implementation of plume rise and its impacts on emissions and air quality modelling. Atmos. Environ. 99, 618-629.

DOI: 10.1016/j.atmosenv.2014.10.029

Guevara M., Tena C., Soret A., Serradell K., Guzmán D., Retama A., Camacho P., Jaimes-Palomera M.y Mediavilla A. (2017). An emission processing system for air quality modelling in the Mexico City metropolitan area: Evaluation and comparison of the MOBILE6.2Mexico and MOVES-Mexico traffic emissions. Sci. Total Environ. 584-585, 882-900.

DOI: $10.1016 /$ j.scitotenv.2017.01.135

INEGI (2011a). Marco Geoestadístico 2010 versión 5.0 A. Instituto Nacional de Estadística y Geografía, México [en línea]. http://www.inegi.org.mx/geo/contenidos/ geoestadistica/m_g_3.aspx. 30/03/2016

INEGI (2011b). Censo de población y vivienda 2010. Instituto Nacional de Estadística y Geografía, México [en línea]. http://www.inegi.org.mx/sistemas/consulta_resultados/ageb_urb2010.aspx?c=28111\&s $=$ est $16 / 0 \overline{3} / 2016$

INEGI (2011c). Conjunto de datos vectoriales de carreteras y vialidades urbanas, edición 1.0 (distribución por entidad federativa). Instituto Nacional de Estadística y Geografía, México [en línea]. http://www.inegi.org. $\mathrm{mx} /$ geo/contenidos/topografia/vectoriales_carreteras. aspx . 16/03/2016

INEGI (2013). Datos vectoriales de uso del suelo y vegetación, serie IV (2007-2010), escala 1:250 000. Instituto Nacional de Estadística y Geografía, México [en línea]. http://www.inegi.org.mx/geo/contenidos/ recnat/usosuelo/ 16 marzo 2016

Jazcilevich A.D., García A.R., Grutter M., Delgado J., Ayala U.D., Lastra M.S., Zuk M., Oropeza R.G., Lents J. y Davis N. (2011). An evaluation of the hybrid car technology for the Mexico mega city. J. Power Sources 196 (13) 5704-5718.

DOI: 10.1016/j.jpowsour.2011.01.076

Mena-Carrasco M., Carmichael G.R., Campbell J.E., Zimmerman D., Tang Y., Adhikary B., D’Allura A., Molina L.T., Zavala M., García A., Flocke F., Campos T., Weinheimer A.J., Shetter R., Apel E., Montzka D.D., Knapp D.J. y Zheng W. (2009). Assessing the regional impacts of Mexico City emissions on air quality and chemistry. Atmos. Chem. Phys. 9 (11) 3731-3743.

DOI: $10.5194 /$ acp-9-3731-2009

Middleton P., Stockwell W.R. y Carter W.P.L. (1990). Aggregation and analysis of volatile organic compound emissions for regional modeling. Atmos. Environ. A-Gen. 24 (5) 1107-1133. DOI: 10.1016/0960-1686(90)90077-Z 
Ortiz L.M. (2005). Sistema para el procesamiento de emisiones atmosféricas (SPEA, v. 1.0.0). Instituto Nacional de Ecología, México, 107 pp.

Qiu X., Zhu Y., Jang C., Lin C.J., Wang S., Fu J., Xie J., Wang J., Ding D. y Long S. (2015). Development of an integrated policy making tool for assessing air quality and human health benefits of air pollution control. Front. Enviro. Sci. Eng. 9 (6) 1056-1065.

DOI: $10.1007 / \mathrm{s} 11783-015-0796-8$

Rew R. y Davis G. (1990). NetCDF: An interface for scientific data access. IEEE Compu. Grap. Appl. 10 (4) 76-82. DOI: $10.1109 / 38.56302$

Ruiz-Suárez L.G. (2015). Estudios de calidad del aire y su impacto en la región centro de méxico, ECAIM. Instituto Nacional de Ecología y Cambio Climático/ CCA-UNAM, México [en línea]. www.inecc.gob.mx/ descargas/calaire/2015_calaire_imp_reg_centro_mex. pdf 7/1272017

SINEA. (2015). Inventario Nacional de Emisiones 2008. Sistema Nacional de Emisiones a la Atmósfera, Secretaría de Medio Ambiente y Recursos Naturales, México [en línea]. http://sinea.semarnat.gob.mx/sinae.php?pr ocess $=$ UkVQT1JURUFET1I $=\&$ categ $=14$ 16/03/2016

Song J., Lei W., Bei N., Zavala M., De Foy B., Volkamer R., Cardenas B., Zheng J., Zhang R. y Molina L.T. (2010). Ozone response to emission changes: A modeling study during the MCMA-2006/MILAGRO campaign. Atmos. Chem. Phys. 10 (8) 3827-3846. DOI: 10.5194/acp-10-3827-2010

Stockwell W.R., Middleton P., Chang J.S. y Xiaoyan T. (1990). The second generation regional acid deposition model chemical mechanism for regional air quality modeling. J. Geophys. Res. 95 (D10) 16343-16367. DOI: 10.1029/JD095iD10p16343

Tie X., Madronich S., Li G., Ying Z., Zhang R., García A.R., Lee-Taylor J. y Liu Y. (2007). Characterizations of chemical oxidants in Mexico City: A regional chemical dynamical model (WRF-Chem) study. Atmos. Environ. 41 (9) 1989-2008.

DOI: 10.1016/j.atmosenv.2006.10.053

Zavala M., Lei W., Molina M.J. y Molina L.T. (2009). Modeled and observed ozone sensitivity to mobilesource emissions in Mexico City. Atmos. Chem. Phys. 9 (1) 39-55. DOI: 10.5194/acp-9-39-2009

Zhang Y. y Dubey M.K. (2009). Comparisons of WRF/ Chem simulated $\mathrm{O}_{3}$ concentrations in Mexico City with ground-based RAMA measurements during the MILAGRO period. Atmos. Environ. 43 (30) 46224631. DOI: $10.1016 /$ j.atmosenv.2009.05.039

Zhang Y., Dubey M.K., Olsen S.C., Zheng J.y Zhang R. (2009). Comparisons of WRF-chem simulations in Mexico City with ground-based RAMA measurements during the 2006-MILAGRO. Atmos. Chem. Phys. 9 (11) 3777-3798. DOI: 10.5194/acp-9-3777-2009 


\begin{tabular}{|c|c|}
\hline $\mathrm{ACT}$ & Acetona \\
\hline AGEB & Área geoestadística básica \\
\hline ALD & Aldehídos \\
\hline ALK3 & Alcanos y no aromáticos con $\mathrm{k}_{\mathrm{oh}}$ de 0.5 a 2.5 \\
\hline ALK4 & Alcanos y no aromáticos con $\mathrm{k}_{\mathrm{oh}}$ de 2.5 a 5 \\
\hline ALK5 & Alcanos y no aromáticos con $\mathrm{k}_{\mathrm{oh}}$ de 5 a 10 \\
\hline ARO1 & Aromáticos con $\mathrm{k}_{\mathrm{oh}}<2$ \\
\hline $\mathrm{ARO} 2$ & Aromáticos con $\mathrm{k}_{\mathrm{oh}}>2$ \\
\hline C-Shell & Intérprete de comandos en modo de texto \\
\hline CCL & Proyección cartográfica cónica conforme de Lambert \\
\hline $\mathrm{CH} 4$ & Metano \\
\hline $\mathrm{CO}$ & Monóxido de carbono \\
\hline $\mathrm{COV}$ & Compuestos orgánicos volátiles \\
\hline CSL & Cresol \\
\hline DiETE & Ditribución espacial, temporal y de especiación \\
\hline ETH & Etano \\
\hline ETHE & Etileno \\
\hline GIS & Sistema de Información Geográfica \\
\hline GLP & Gas LP \\
\hline GNC & Gas natural comprimido \\
\hline $\mathrm{GSO}_{4}$ & Partículas primarias de sulfato \\
\hline $\mathrm{HC} 3$ & Propano \\
\hline $\mathrm{HC} 5$ & Alcanos $\left(\mathrm{k}_{\mathrm{oh}}\right.$ de 0.5 a 1.0$)$ \\
\hline $\mathrm{HC} 8$ & Alcanos $\left(\mathrm{k}_{\mathrm{oh}}\right.$ de 1 a 2$)$ \\
\hline $\mathrm{HCHO}$ & Formaldehído \\
\hline HDDV & Heavy duty diesel vehicle \\
\hline HDGV & Heavy duty gasoline vehicle \\
\hline HKET & Hidroxi cetona \\
\hline INEM & Inventario Nacional de Emisiones de México \\
\hline ISO & Isopreno \\
\hline ITRF92 & Datum international terrestrial reference frame 92 \\
\hline LDDT & Light duty diesel truck \\
\hline LDGT & Light duty gasoline truck \\
\hline LDGV & Light duty gasoline vehicle \\
\hline Ma & Masa del inventario distribuida en el área \\
\hline MCA & Modelo de calidad del aire \\
\hline Mo & Masa original del inventario \\
\hline Mt & Masa del inventario distribuida espacial y temporalmente \\
\hline $\mathrm{NO}$ & Óxido de nitrógeno \\
\hline $\mathrm{NO}_{2}$ & Dióxido de nitrógeno \\
\hline NROG & Compuestos orgánicos no reactivos \\
\hline OTHER & Otros aerosoles \\
\hline OLE2 & Alquenos con $\mathrm{k}_{\mathrm{oh}}>7$ \\
\hline OLI & Alquenos internos \\
\hline OLT & Alquenos terminales \\
\hline PEC & Partículas carbono elemental \\
\hline PHEN & Fenol \\
\hline $\mathrm{PNO}_{3}$ & Partículas primarias de nitrato \\
\hline POA & Primarias de aerosoles orgánicos \\
\hline RACM & Regional atmospheric chemistry mechanism \\
\hline RADM2 & Regional Atmospheric Deposition Model, version 2 \\
\hline $\mathrm{RCHO}$ & Aldehídos agrupados $\mathrm{C} 3+$ \\
\hline SAPRC99 & Statewide Air Pollution Research Center chemical mechanism \\
\hline $\mathrm{SCC}$ & Source clasification code \\
\hline SEMARNAT & Secretaría del Medio Ambiente y Recursos Naturales \\
\hline SINEA & Subsistema del Inventario Nacional de Emisiones a la Atmósfera de México de entidades federativas y municipios \\
\hline $\mathrm{SO}_{2}$ & Dióxido de azufre \\
\hline TOL & Tolueno \\
\hline WRF & Weather Research and Forecast model \\
\hline XYL & Xilenos \\
\hline XYM & Meta xileno \\
\hline
\end{tabular}

